\title{
Ornithine transcarbamylase downregulation is associated with poor prognosis in hepatocellular carcinoma
}

\author{
LIN HE $^{1}$, XUEFEI CAI ${ }^{1}$, SHENGTAO CHENG ${ }^{1}$, HONGZHONG ZHOU $^{1}$, ZHENZHEN ZHANG $^{2}$,

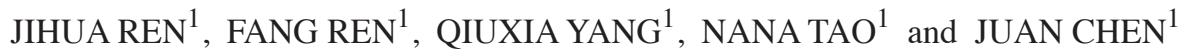 \\ ${ }^{1}$ Key Laboratory of Molecular Biology of Infectious Diseases Designated by The Chinese Ministry of Education, \\ Chongqing Medical University, Chongqing 400016; ${ }^{2}$ Department of Infectious Diseases, \\ The Children's Hospital of Chongqing Medical University, Chongqing 400014, P.R. China
}

Received April 9, 2018; Accepted February 22, 2019

DOI: $10.3892 / \mathrm{ol} .2019 .10174$

\begin{abstract}
Hepatocellular carcinoma (HCC) is one of the leading causes of cancer-associated mortalities worldwide. The role of ornithine transcarbamylase (OTC) in HCC remains unclear. In the present study, the expression of OTC in HCC was analyzed based on datasets from the Gene Expression Omnibus database of the National Center for Biotechnology Information and further confirmed by immunohistochemistry, western blotting analysis and reverse transcription-quantitative polymerase chain reaction assays on clinical samples and cell lines. Furthermore, the associations between OTC expression and clinicopathological parameters as well as clinical outcome, including the overall and disease-free survival rates were analyzed. Finally, the effect of OTC on HCC cells was measured using proliferation, bromodeoxyuridine and colony-formation assays. Lower OTC expression was observed in HCC cells and tissues compared with primary human hepatocytes. Further investigation demonstrated that low expression of OTC in HCC was associated with larger tumor size and advanced grade. A Kaplan-Meier analysis revealed that patients with lower levels of OTC exhibited shorter overall and disease-free survival times. Notably, OTC silencing with RNA interference facilitated cell proliferation in HCC SK-Hep-1 and Huh-7 cells. However, overexpression of OTC led to inhibition of cell proliferation. In conclusion, the present study identified a novel role of OTC in HCC development, providing a potential novel therapeutic target for this disease.
\end{abstract}

Correspondence to: Professor Juan Chen, The Key Laboratory of Molecular Biology of Infectious Diseases Designated by The Chinese Ministry of Education, Chongqing Medical University, 1 Medical College Avenue, Chongqing 400016, P.R. China E-mail: chenjuan2014@cqmu.edu.cn

Key words: hepatocellular carcinoma, ornithine transcarbamylase, tumor suppressor, prognosis

\section{Introduction}

Hepatocellular carcinoma (HCC) accounts for 70-90\% of primary liver cancer, as estimated in 2012 (1), with $>500,000$ patients worldwide being diagnosed with hepatocellular carcinoma, as estimated in 2011 (2). Liver cancer has been predicted to be the sixth most commonly diagnosed type of cancer and the fourth leading cause of cancer-associated mortality worldwide in 2018, with $\sim 841,000$ new cases and 782,000 cases of cancer-associated mortality annually (3). Apart from surgery, multiple treatment options are available for HCC treatment, including resection, ablation, transplantation, chemoembolization and systemic targeted agents, such as sorafenib (4). However, resistance to chemotherapeutic drugs, cancer recurrence and early metastasis remain major obstacles in clinical treatment (5). Therefore, improved understanding of the molecular mechanisms in HCC is required.

Ornithine transcarbamylase (OTC) is a liver and intestinal mucosa-located, intramitochondrial, rate-limiting enzyme in the urea cycle. It catalyzes the reaction that converts ornithine and carbamoyl phosphate into citrulline, which is the second step of the urea cycle, to detoxify the ammonia produced from amino acid catabolism (6). Defects in the urea cycle can lead to high blood ammonia concentration, which can cause several physiological disorders, including anorexia, cerebral edema, coma, seizure, delayed growth and development, intellectual disabilities and even loss of life $(7,8)$. Several studies have suggested that accumulated ammonia resulting from OTC deficiency causes chronic liver damage, which is a potential risk factor of HCC (9-11). Furthermore, increased liver fibrosis in OTC-knockout Het mice compared with their wild-type littermates has been confirmed (12). However, studies on the effect of OTC in HCC are limited.

The present study aimed to identify whether OTC serves a role in HCC progression, and to investigate its biological function. The present study revealed that OTC downregulation is a characteristic molecular alteration and a reliable prognostic factor for the survival of patients with HCC. Notably, the present study demonstrated anti-growth effects of OTC in HCC cells for the first time. The present study suggested that OTC may be investigated as a novel HCC therapeutic. 


\section{Materials and methods}

Plasmids and antibodies. Small interfering RNAs (siRNAs) targeting OTC (siOTC-1 and siOTC-2) and negative control (siCont) were purchased from Guangzhou RiboBio Co., Ltd. (Guangzhou, China). The pCMV6-XL4-OTC expression plasmid and the empty vector pCMV6-XL4 were obtained from OriGene Technologies, Inc. (Rockville, MD, USA). The antibody for OTC was purchased from Sigma-Aldrich (Merck KGaA, Darmstadt, Germany; cat. no. \#AV41766) and the antibody for GAPDH was from Santa Cruz Biotechnology, Inc. (Dallas, TX, USA; cat. no. sc-365062).

Cell culture. Human liver cancer cell lines SK-Hep-1, HepG2, PLC/PRF/5 and Huh-7 were obtained from the American Type Culture Collection (Manassas, VA, USA). The immortalized hepatocyte MIHA cell line was obtained from Professor Ben C. B. Ko (The Hong Kong Polytechnic University, Hong Kong, SAR, China). Primary human hepatocytes (PHHs) were purchased from ScienCell Research Laboratories, Inc. (San Diego, CA, USA). The liver cancer and MIHA cells were cultured in Dulbecco's modified Eagle's medium supplemented with $10 \%$ fetal bovine serum (Gibco; Thermo Fisher Scientific, Inc., Waltham, MA, USA). The PHHs were cultured in hepatocyte medium (ScienCell Research Laboratories, Inc.). All cells were maintained in a humidified atmosphere at $37^{\circ} \mathrm{C}$ in $5 \% \mathrm{CO}_{2}$.

Cell transfection. All siRNAs and plasmids were transfected into cells using Lipofectamine ${ }^{\circledR} 3000$ (Invitrogen; Thermo Fisher Scientific, Inc.), according to the manufacturer's protocol. Specifically, cells were seeded into 6-well-plates the day before transfection and were grown to 70-90\% confluency prior to transfection. For OTC knockdown analysis, $0.1 \mathrm{nmol}$ siOTC-1 or siOTC-2, or siCont (final concentration, $50 \mu \mathrm{M}$ ) and Opti-MEM (Gibco; Thermo Fisher Scientific, Inc.) were mixed and incubated for $10 \mathrm{~min}$, and subsequently added to SK-Hep-1 and Huh-7 cells. For OTC overexpression analysis, $2 \mu \mathrm{g}$ plasmids (pCMV6-XL4-OTC or pCMV6-XL4), Opti-MEM (Gibco; Thermo Fisher Scientific, Inc.) and Lipofectamine $^{\circledR} 3000$ were mixed and incubated for $10 \mathrm{~min}$, and then added to SK-Hep-1 and Huh-7 cells. After $6 \mathrm{~h}$ of incubation, the supernatant was replaced with fresh medium. The transfection efficiency was monitored by western blot analysis after 3 days of transfection. The bromodeoxyuridine assays were conducted 3 days after transfection. For the colony formation assay, colonies were stained 12 days after transfection. For the proliferation assay, cell numbers were counted daily between the second and fifth day after transfection.

Tissue specimens. A total of 42 pairs of primary HCC and matched adjacent histologically normal liver tissue samples were collected from newly-diagnosed patients with HCC at the First Affiliated Hospital of Chongqing Medical University (Chongqing, China) between December 2016 and December 2017. Of these patients, $36(85.7 \%)$ were male and six $(14.4 \%)$ were female. Patient ages ranged between 22 and 77 years $(56.88 \pm 13.404$ years $)$. A total of 10 parameters that may be prognostic in HCC were assessed by statistical analyses and summarized in Table I. The following inclusion criteria were used: i) Patient had received no radiotherapy or chemotherapy prior to surgery; and ii) patient exhibited pathologically defined primary tumors according to the International Union Against Cancer guidelines (13). Histological grading was performed according to the Edmondson-Steiner criteria (14). Following surgical resection, tumor diameter was measured and the samples were immediately snap frozen in liquid nitrogen and stored at $-80^{\circ} \mathrm{C}$. Relevant clinical data were collected retrospectively. Additionally, paraffin-embedded sections of 15 paired samples from patients with HCC, which were not included in the aforementioned group of patients, were obtained for immunohistochemical analysis from the Pathology Department of First Affiliated Hospital, Chongqing Medical University. Of the 15 patients, 10 (66.7\%) were male and $5(33.3 \%)$ were female. Patient ages ranged between 39 and 73 years (mean \pm standard deviation, 62.67 \pm 9.401 years). Written informed consent was obtained from each patient recruited to the study, which was approved by the Clinical Research Ethics Committee of Chongqing Medical University.

Datasets. Gene expression profiles were downloaded using $\mathrm{R}$ version 3.2.4 software ( $\mathrm{R}$ Foundation for Statistical Computing, Vienna, Austria) from the Gene Expression Omnibus database (dataset accession no. GSE14520) (15). RNA-sequencing and detailed clinicopathological data for patients with $\mathrm{HCC}$ were obtained from The Cancer Genome Atlas (TCGA; project ID: TCGA-LIHC; https://portal.gdc. cancer.gov/projects/TCGA-LIHC). The data from TCGA were $\log _{10}$ transformed and analyzed using SPSS version 19.0 software (IBM Corporation, Chicago, IL, USA).

Reverse transcription-quantitative polymerase chain reaction $(R T-q P C R)$. Total RNA of all cell lines and liver tissues collected in the present study was extracted using TRIzol reagent (Tiangen Biotech Co., Ltd., Beijing, China). cDNA was synthesized from $1 \mu \mathrm{g}$ total RNA using an FastQuant RT kit (Tiangen Biotech Co., Ltd.), according to the manufacturer's protocol. qPCR was conducted using a CFX Connect Real-Time system (Bio-Rad Laboratories, Inc., Hercules, CA, USA). Thermocycling conditions were as follows: Initial denaturation at $95^{\circ} \mathrm{C}$ for $2 \mathrm{~min}$, and $20 \mathrm{sec}$ denaturing at $94^{\circ} \mathrm{C}, 20 \mathrm{sec}$ annealing at $60^{\circ} \mathrm{C}$ and $20 \mathrm{sec}$ extension at $72^{\circ} \mathrm{C}$ for 35 cycles, followed by $0.5^{\circ} \mathrm{C}$ every $5 \mathrm{sec}$ from 65 to $95^{\circ} \mathrm{C}$ for melting curve analysis. FastStart Universal SYBR Green Master Mix (Roche Diagnostics, Indianapolis, IN, USA) was used. The sequences of primers were as follows: OTC forward, 5'-TGG CTGATTACCTCACGCTC-3' and reverse, 5'-TTCTTCTGG CTTTCTGGGCAA-3'; $\beta$-actin forward, 5'-CTCTTCCAG CCTTCCTTCCT-3' and reverse, 5'-AGCACTGTGTTGGCG TACAG-3'. The experiments were performed 3 times. The relative OTC mRNA expression levels were determined with $\beta$-actin as an endogenous control, using the $2^{-\Delta \Delta \mathrm{Cq}}$ method (16).

Western blot analysis. The cells were harvested and lysed with radioimmunoprecipitation assay lysis buffer supplemented with a protease inhibitor cocktail (Roche Diagnostics). Protein quantification was conducted using the BCA assay (Pierce; Thermo Fisher Scientific, Inc.). Total proteins (30 $\mu \mathrm{g} /$ lane) were separated using 10\% SDS-PAGE and transferred to polyvinylidene difluoride membranes (GE Healthcare Life Sciences, Little Chalfont, UK). The membranes were blocked with 5\% 
Table I. Patient clinicopathological features and tumor OTC expression.

\begin{tabular}{|c|c|c|c|c|}
\hline \multirow[b]{2}{*}{ Characteristic } & \multirow[b]{2}{*}{$\mathrm{n}$} & \multicolumn{2}{|c|}{ OTC expression ${ }^{\mathrm{a}}$} & \multirow[b]{2}{*}{ P-value } \\
\hline & & Low & High & \\
\hline Sex & & & & 1.00 \\
\hline Female & 6 & 4 & 2 & \\
\hline Male & 36 & 26 & 10 & \\
\hline Age, years $($ mean $\pm S D)$ & & $55.5 \pm 14.6$ & $60.4 \pm 11.3$ & 0.29 \\
\hline ALT, IU/1 & & & & 0.33 \\
\hline$\leq 40$ & 23 & 15 & 8 & \\
\hline$>40$ & 19 & 15 & 4 & \\
\hline $\mathrm{CEA}, \mathrm{ng} / \mathrm{ml}$ & & & & 0.39 \\
\hline$\leq 5$ & 35 & 26 & 9 & \\
\hline$>5$ & 7 & 4 & 3 & \\
\hline $\mathrm{AFP}, \mathrm{ng} / \mathrm{ml}$ & & & & 0.14 \\
\hline$\leq 20$ & 24 & 15 & 9 & \\
\hline$>20$ & 18 & 15 & 3 & \\
\hline Tumor diameter, $\mathrm{cm}$ & & & & $0.01^{\mathrm{b}}$ \\
\hline$\leq 3$ & 7 & 2 & 5 & \\
\hline$>3$ & 35 & 28 & 7 & \\
\hline Multiple tumors & & & & 0.39 \\
\hline No & 35 & 26 & 9 & \\
\hline Yes & 7 & 4 & 3 & \\
\hline Tumor grade & & & & $0.02^{\mathrm{b}}$ \\
\hline 1 & 8 & 3 & 5 & \\
\hline 2 & 27 & 20 & 7 & \\
\hline 3 & 7 & 7 & 0 & \\
\hline Vascular invasion & & & & 0.32 \\
\hline No & 26 & 17 & 9 & \\
\hline Yes & 16 & 13 & 3 & \\
\hline Cirrhosis & & & & 0.72 \\
\hline No & 12 & 8 & 4 & \\
\hline Yes & 30 & 22 & 8 & \\
\hline
\end{tabular}

${ }^{\mathrm{a}}$ OTC protein expression level in tumor tissue relative to the adjacent non-tumor liver sample, as determined by western blot analysis, values expressed as number of patients, unless otherwise specified. ${ }^{\mathrm{b}} \mathrm{P}<0.05$. OTC, ornithine transcarbamylase; $\mathrm{SD}$, standard deviation; ALT, alanine aminotransferase; CEA, carcinoembryonic antigen; AFP, $\alpha$-fetoprotein.

skimmed milk for $1 \mathrm{~h}$ at room temperature, and incubated with OTC primary antibody (1:1,000 dilution) or GAPDH (1:5,000 dilution) primary antibody at $4{ }^{\circ} \mathrm{C}$ overnight. They were subsequently incubated with horseradish peroxidase-conjugated anti-rabbit (1:3,000 dilution; cat. no. NA934; GE Healthcare Life Sciences) or anti-mouse IgG secondary antibodies (1:5,000 dilution; cat. no. sc-2005; Santa Cruz Biotechnology, Inc.) at room temperature for $2 \mathrm{~h}$. The blots were developed with ECL western blotting analysis reagents (Merck KGaA) and the signal intensities were quantified using ImageJ version 1.4 software (National Institutes of Health, Bethesda, MD, USA).
Immunohistochemistry (IHC). Immunohistochemistry (IHC) was performed on paraffin-embedded sections. Sections $(5-\mu \mathrm{m})$ of 15 paired samples from patients with HCC were obtained from the Pathology Department of First Affiliated Hospital, Chongqing Medical University. The tissue sections were dewaxed with xylene, rehydrated through an ethanol gradient into water and microwave-heated in sodium citrate buffer (10 mM, pH 6.0) for antigen retrieval. Endogenous peroxidase activity was quenched with $3 \% \mathrm{H}_{2} \mathrm{O}_{2}$, and the sections were then incubated with $10 \%$ normal goat nonimmune serum (Fuzhou Maixin Biotech Co., Ltd., Fuzhou, China) for $30 \mathrm{~min}$ at room temperature to block the nonspecific antibody binding. Subsequently, the slides were incubated with OTC antibody at a 1:50 dilution at $4^{\circ} \mathrm{C}$ overnight. The following day, the sections were incubated with biotinylated secondary antibody (undiluted; cat. no. KIT-9710; Fuzhou Maixin Biotech Co., Ltd.) and combined with horseradish peroxidase-labeled streptavidin. DAB staining was used for detecting immunoreactivity (Dako; Agilent Technologies, Inc., Santa Clara, CA, USA). Counterstaining was performed using hematoxylin for $2 \mathrm{~min}$ at room temperature. Negative control sections were prepared without primary antibody to determine the specificity of the staining. The scoring of OTC was carried out by two independent pathologists using a light microscope, according to the proportion of tumor cells with positive staining (negative, none; weak, <10\%; moderate, $10-50 \%$; strong, $>50 \%$ ), as previously described (17). No and weak staining were defined as OTC negative, and moderate and strong staining were defined as OTC positive. Images were captured using an Olympus microscope equipped with a Olympus DP71 digital camera (magnification, x200; Olympus Corporation, Tokyo, Japan).

Proliferation and bromodeoxyuridine assays. Cell proliferation in response to OTC silencing or overexpression was determined by a trypan blue exclusion assay (Thermo Fisher Scientific, Inc.). DNA synthesis was examined using a Click-iT ${ }^{\circledR}$ EdU Imaging kit (Invitrogen; Thermo Fisher Scientific, Inc.), according to the manufacturer's protocols.

Colony-formation assay. Cells transfected with plasmids (pCMV6-XL4-OTC and pCMV6-XL4) and siRNAs (siOTC-1, siOTC-2 and siCont) were re-seeded in 12-well plates and incubated at $37^{\circ} \mathrm{C}$ for 12 days, with the medium being changed every 3 days. Images and metrics of newly formed colonies were obtained by using IncuCyte ${ }^{\circledR}$ (model: CCL-170T-8; Essen Bioscience, Ann Arbor, MI, USA). Each experiment was repeated independently 3 times.

Statistical analysis. The difference between two groups was assessed using two-tailed Student's t-test. OTC expression in HCC and adjacent non-tumor liver tissue samples was compared using paired two-tailed Student's t-test. Differences in OTC positive rates between HCC and adjacent non-tumor liver tissues, as examined by IHC, were evaluated by Fisher's exact test. One-way analysis of variance followed by Dunnet's post hoc test was performed for comparisons among multiple groups. Associations between OTC expression and clinicopathological parameters were evaluated using a nonparametric $\chi^{2}$ test. The survival rate analysis was performed using the 
A

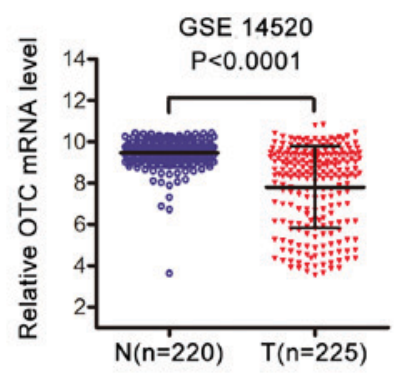

C

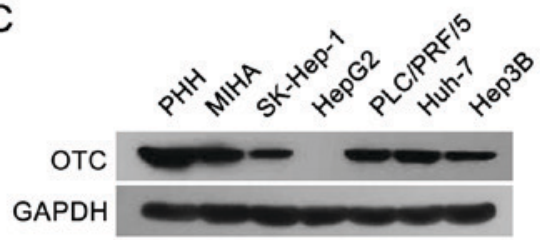

F

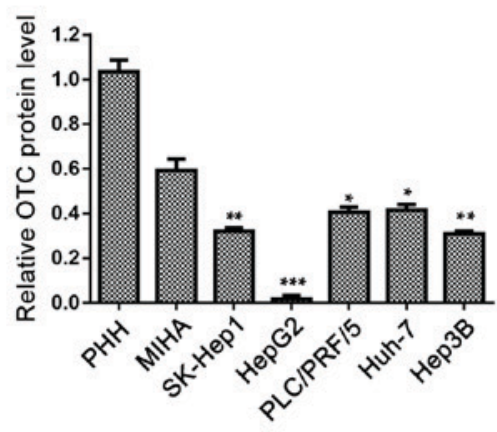

B

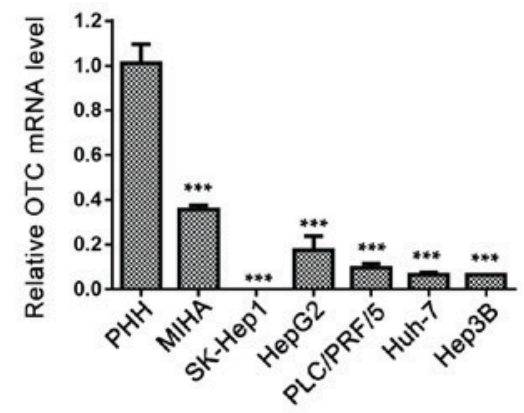

D

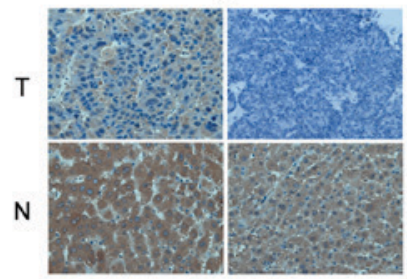

E

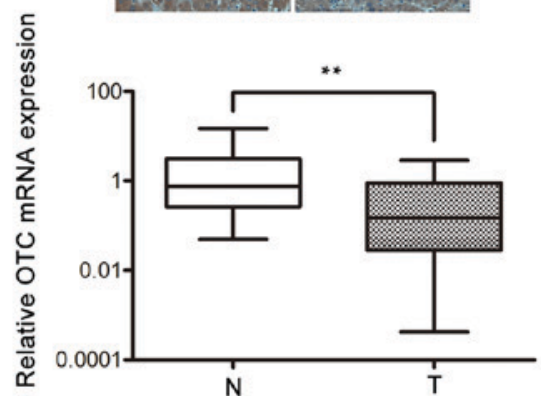

G

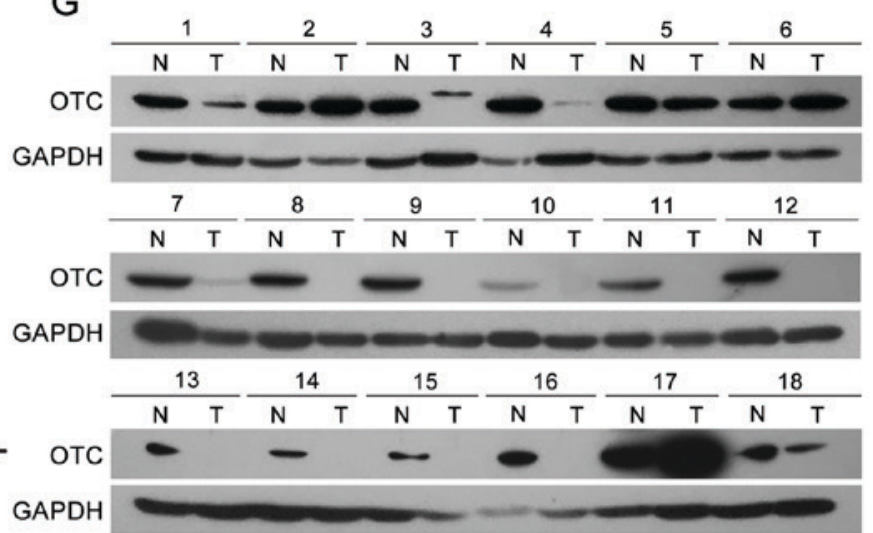

Figure 1. OTC expression is significantly downregulated in HCC. (A) The relative level of OTC mRNA in HCC T and N samples. The microarray data were obtained from the Gene Expression Omnibus database (dataset GSE14520). The central line represents the mean and the error bars indicate the standard deviation. The average relative OTC mRNA levels were compared using a two-tailed unpaired Student's t-test. ${ }^{* * *} \mathrm{P}<0.0001$. OTC (B) mRNA and (C) protein expression in PHHs, 5 liver cancer cell lines and the immortalized liver MIHA cells. GAPDH was used as a loading control for the western blot analysis and $\beta$-actin was used as a reference gene for in the reverse transcription-quantitative polymerase chain reactions. The data are presented as the mean \pm standard deviation of 3 independent experiments. ${ }^{* * *} \mathrm{P}<0.001$. (D) Representative immunohistochemistry images of OTC expression in HCC T (top panel) and adjacent $\mathrm{N}$ (bottom panel) samples (original magnification, $\mathrm{x} 400$ ). Upper left, low expression; upper right, negative staining; lower left, strongly positive expression; lower right, moderate levels of staining. Quantitative analysis of OTC (E) mRNA and (F) protein levels in 42 paired clinical HCC tissue samples. The central line represents the mean and the error bars indicate the standard deviation. Two-tailed paired Student's t-test was used to compare the groups. ${ }^{*} \mathrm{P}<0.05 ;{ }^{* * *} \mathrm{P}<0.01$ and ${ }^{* * * *} \mathrm{P}<0.001$. (G) Western blot analysis of OTC (39 kDa) in 18 paired clinical HCC T and adjacent N samples. GAPDH was used as a loading control. OTC, ornithine transcarbamylase; HCC, hepatocellular carcinoma; T, tumor tissue; N, non-tumor tissue; PHH, primary human hepatocyte

Kaplan-Meier method and equivalences of the survival curves were tested using log-rank statistics. All statistical analyses were performed using SPSS version 19.0 software. Differences were considered statistically significant when $\mathrm{P}<0.05$.

\section{Results}

OTC expression is significantly downregulated in HCC. Data from $225 \mathrm{HCC}$ tumor and 220 non-tumor tissue samples from the Gene Expression Omnibus database of the National Center for Biotechnology Information (dataset accession no. GSE14520; http://www.ncbi.nlm.nih.gov/geo/) (15) were used for analysis. The results revealed a significantly decreased OTC expression in the HCC tumor specimens compared with that in the non-tumor samples $(\mathrm{P}<0.0001$; Fig. $1 \mathrm{~A})$. To verify the downregulation of OTC in HCC, its expression in PHHs, immortalized MIHA hepatocytes and liver cancer cells (Huh-7, HepG2, Hep3B, PLC/PRF/5 and SK-Hep-1) was 
determined using RT-qPCR and western blot analyses. The RT-qPCR analysis revealed that OTC mRNA expression was significantly lower in the liver cancer cells compared with that in PHHs (Fig. 1B). In addition, the OTC protein levels were lower in the liver cancer cell lines compared with those in PHHs, as detected by western blotting (Fig. 1C). Similar to HCC cells, MIHA cells exhibited lower OTC expression compared with PHH cells. As numerous genes identified to be dysregulated were associated with cell immortalization and transformation $(18,19)$, it is possible that immortalization contributed to the downregulation of OTC in MIHA cells.

Subsequently, OTC protein expression was investigated in 15 HCC tissue samples obtained from the Pathology Department of First Affiliated Hospital, Chongqing Medical University and their respective adjacent non-tumor liver samples via IHC (Fig. 1D). The findings revealed that, in the 15 cases examined, all the adjacent non-tumor liver tissues exhibited positive (strong or moderate) OTC expression. However, positive expression was detected in only 4 of the 15 HCC tissues (26.7\%) (Table SI). The levels of OTC were further examined in 42 pairs of clinical HCC specimens by RT-qPCR and western blotting. The results indicated that OTC mRNA was downregulated on average in the HCC tissues compared with their adjacent non-tumor samples $(\mathrm{P}=0.0006$; Fig. 1E). Western blot analysis indicated that 30/42 HCC tissues (71.4\%) exhibited lower OTC expression compared with adjacent non-tumor tissues. Of these $30 \mathrm{HCC}$ tissues, 14 exhibited undetectable OTC protein expression. The average OTC protein level in the $42 \mathrm{HCC}$ tissues was significantly lower compared with the average OTC protein level in the 42 adjacent non-tumor samples, as semi-quantified by ImageJ software. ( $\mathrm{P}=0.0404$; Fig. $1 \mathrm{~F}$ and $\mathrm{G})$. Taken together, the present results suggest that OTC expression is suppressed in HCC tissues and cells.

The association between OTC expression and clinicopathological parameters. The association between OTC expression and the clinicopathological features of the patients with HCC was examined in the 42 cases included in this study. The patients were classified into two groups based on the OTC protein level (cases where the protein level in the tumor tissue relative to the adjacent non-tumor tissue sample was $<1.0$ were classified as low-expression; the rest were classified as high-expression). The findings revealed low expression in 30 of the 42 cases (71.4\%) and high expression in 12 cases (28.6\%) (Table I). Pearson's $\chi^{2}$ test revealed that lower tumor OTC levels were associated with a larger tumor size $(\mathrm{P}=0.01)$ and higher grade $(\mathrm{P}=0.02)$. No significant association was observed between OTC levels and patient age or sex, or other clinical parameters, including cirrhosis, vascular invasion, multiple tumors and the serological predictor of liver damage, alanine aminotransferase. In addition, the tumor OTC levels were evaluated according to the tumor stage in data obtained from the TCGA database (http//gdc.cancer.gov/). As demonstrated in Fig. 2A, the OTC mRNA levels in patients with early stage tumors (stage I) was higher than those with stage II $(\mathrm{P}=0.0029)$ or III $(\mathrm{P}=0.0002)$ tumors. To further confirm that low OTC levels indicate poor HCC prognosis, the 5-year overall survival times of $373 \mathrm{HCC}$ cases and disease-free survival times of 313 cases from TCGA database were analyzed using the Kaplan-Meier method.
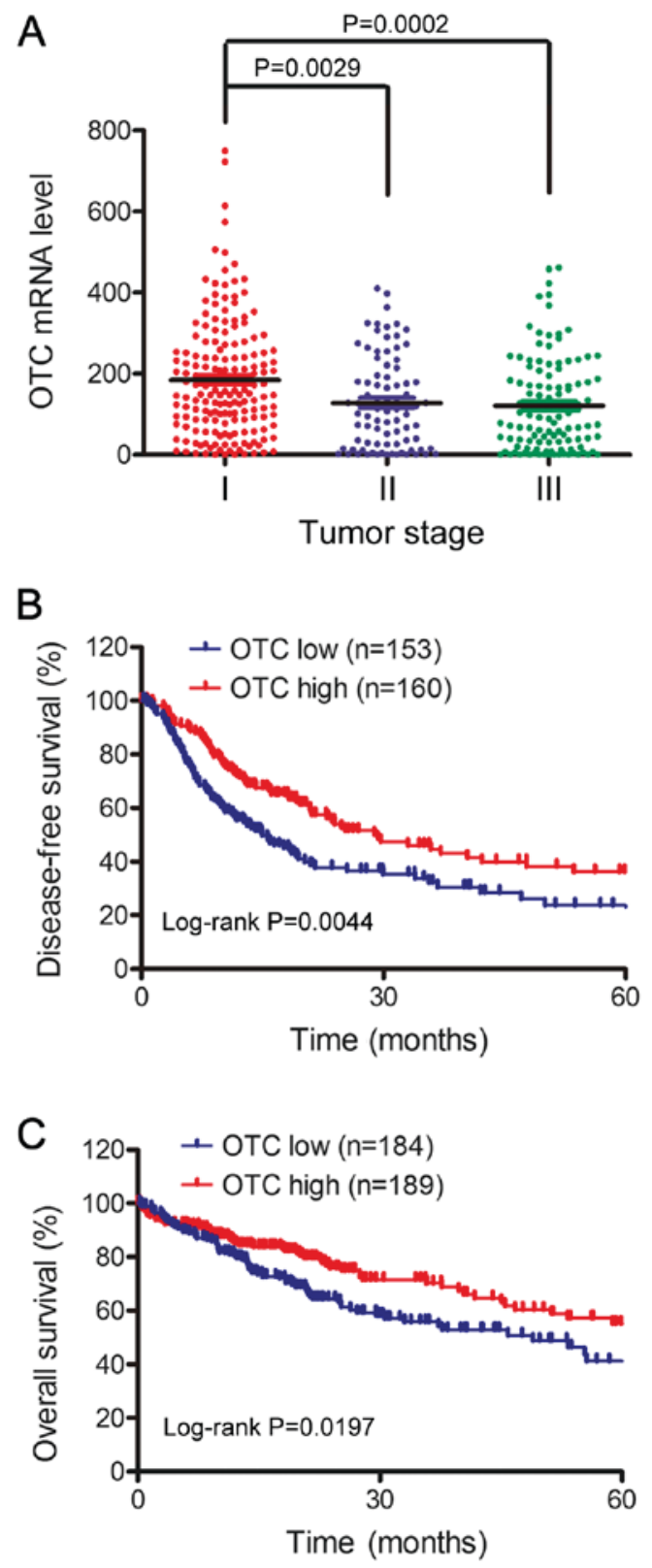

Figure 2. OTC expression and clinicopathological parameters. (A) Relative OTC expression in HCC samples from TCGA database, according to tumor stage. Stage I, $n=172$; stage II, $n=85$; and stage III, $n=114$. The association between OTC expression levels and (B) disease-free survival or (C) overall survival rate in a cohort from TCGA cohort, determined by Kaplan-Meier survival analysis and a log-rank (Mantel-Cox) test. OTC, ornithine transcarbamylase; TCGA, The Cancer Genome Atlas.

Patients with low OTC expression exhibited significantly shorter disease-free survival ( $\mathrm{P}=0.0044$; Fig. 2B) and 5-year overall survival rates $(\mathrm{P}=0.0197$; Fig. $2 \mathrm{C})$ compared with those with high expression. Together, these results demonstrate that low OTC levels are associated with tumor progression and poor survival rates, making this factor a potential novel biomarker for patients with HCC.

OTC overexpression inhibits HCC cell proliferation. To explore the functional role of OTC in HCC, OTC was overexpressed in Huh-7 and SK-Hep-1 cells by transient transfection and verified by western blotting (Fig. 3A). The effects of OTC overexpression on the cells were assessed by cell proliferation 
A



C

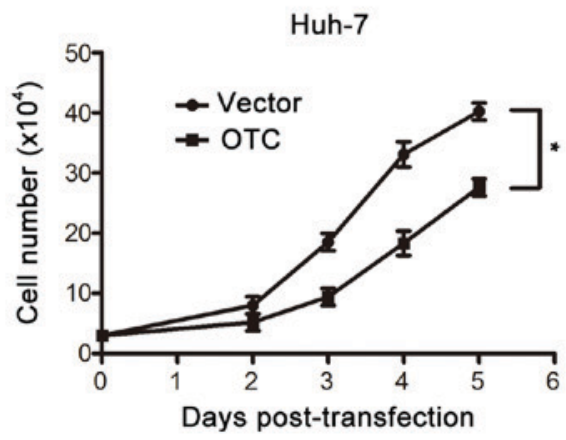

E

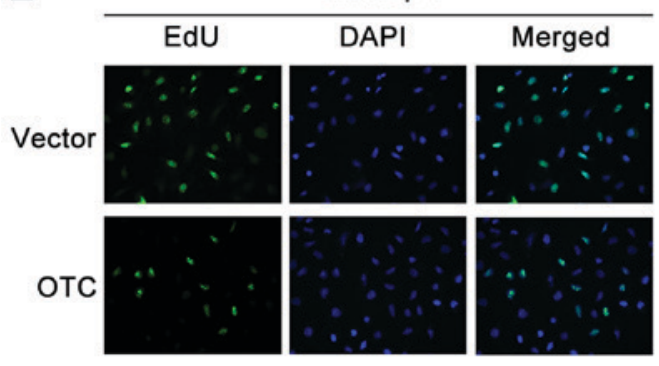

$\mathrm{F}$

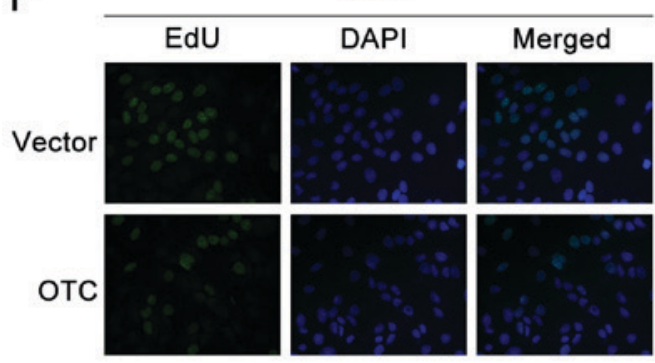

B

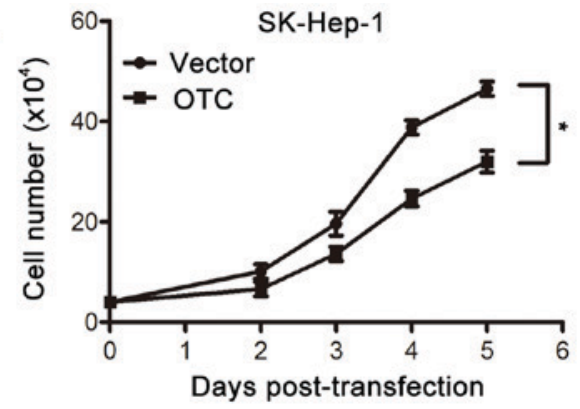

D
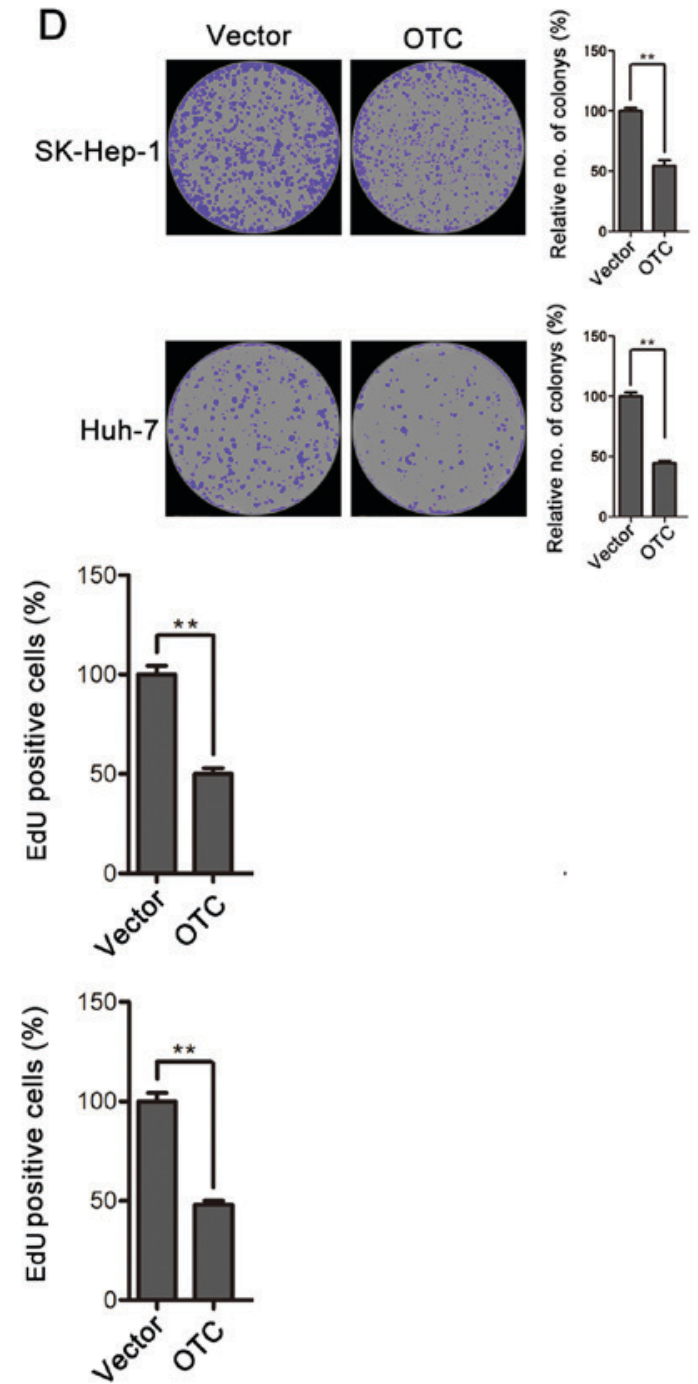

Figure 3. OTC overexpression inhibits HCC cell proliferation. (A) OTC overexpression in HCC SK-Hep-1 and Huh-7 cells, detected by western blot analysis. The cells were transfected with the OTC expression plasmid (pCMV6-XL4-OTC) or the corresponding empty vector (pCMV6-XL4) and harvested 3 days after transfection. Upregulated OTC led to an inhibited proliferation rate in (B) SK-Hep-1 and (C) Huh-7 cells. The cells were counted at the indicated time points using a trypan blue exclusion assay. The data are presented as the mean \pm standard deviation of 3 independent experiments and the groups were compared using two-way analysis of variance. ${ }^{*} \mathrm{P}<0.05$. (D) OTC overexpression resulted in inhibited colony formation. SK-Hep-1 and Huh-7 cells transfected with the OTC expression plasmid or the corresponding empty vector were cultured for 12 days, images were captured, and newly formed colonies in each well were counted and expressed as a percentage relative to the control group. The data are presented as the mean \pm standard deviation of 3 independent experiments, and the groups were compared using two-tailed unpaired Student's t-test. ${ }^{* *} \mathrm{P}<0.01$. DNA synthesis in (E) SK-Hep-1 and (F) Huh-7 cells was determined 3 days after transfection. Original magnification, $\mathrm{x} 400$. The quantification of EdU-positive cells was conducted macroscopically and expressed as a percentage relative to the control group. The data are presented as the mean \pm standard deviation of 3 independent experiments and the groups were compared using two-tailed unpaired Student's t-test. "P<0.01. OTC, ornithine transcarbamylase; HCC, hepatocellular carcinoma.

and colony-formation assays. The upregulation of OTC significantly decreased the proliferation rates of Huh-7 and SK-Hep-1 cells (Fig. 3B and C). Furthermore, decreased numbers and sizes of SK-Hep-1 and Huh-7 cell colonies were observed in the OTC overexpression groups compared with the control groups, which were transfected with vector plasmid (Fig. 3D). Finally, OTC overexpression led to an inhibition of DNA synthesis, as revealed by EdU staining (Fig. 3E and F). Collectively, these results demonstrate that OTC overexpression serves an inhibitory role in cell proliferation and colony-formation in HCC cells. 
A

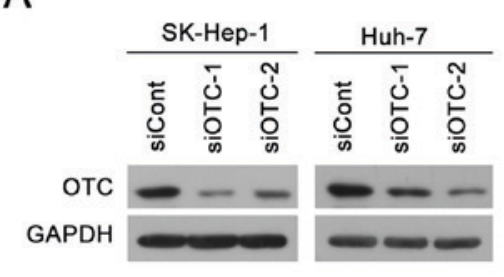

C

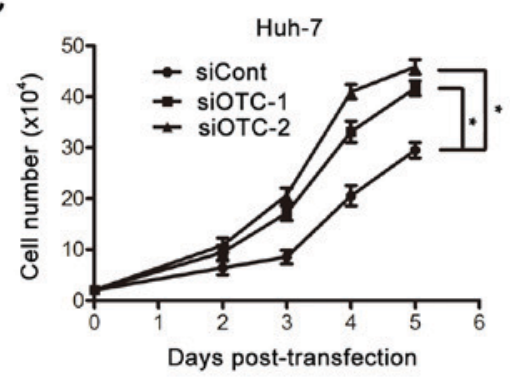

E

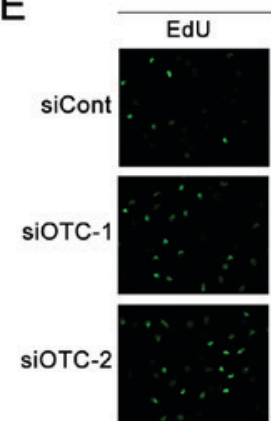

F

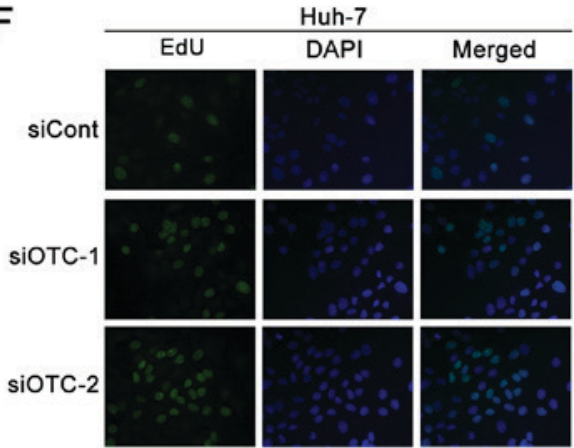

B

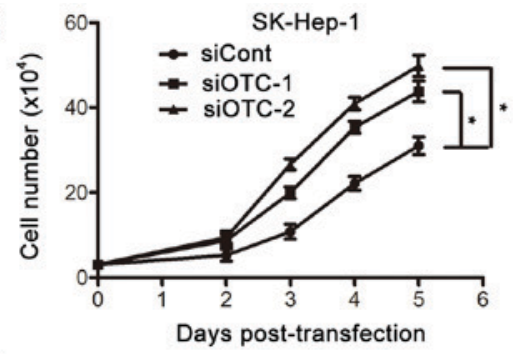

D
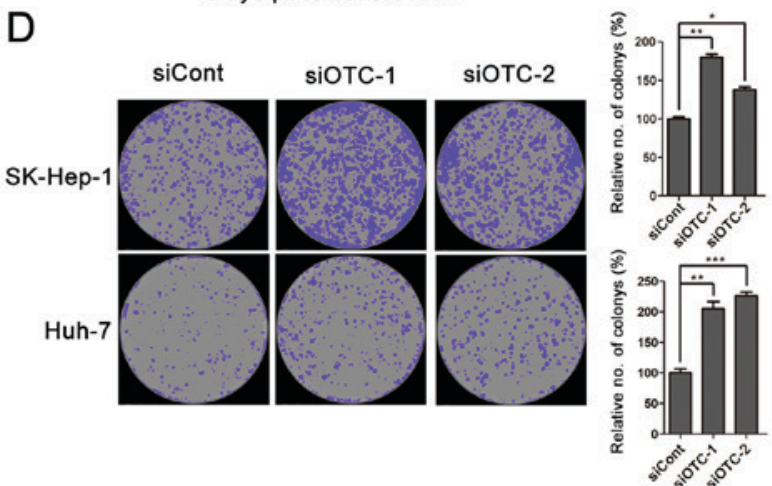
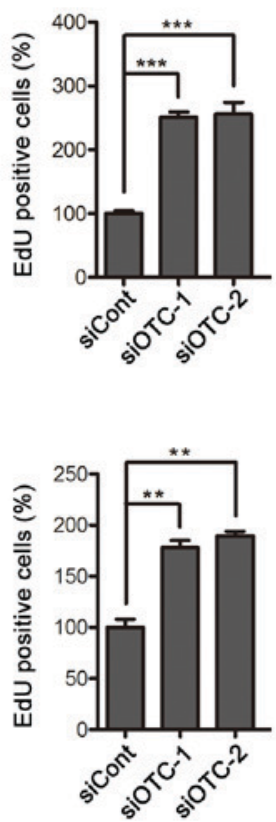

Figure 4. OTC silencing facilitates HCC cell proliferation. (A) The efficiency of OTC gene silencing was evaluated by western blotting. siRNAs siOTC-1, siOTC-2 or siCont were transfected into HCC SK-Hep-1 and Huh-7 cells. The cells were harvested 3 days after transfection. OTC silencing resulted in enhanced proliferation of (B) SK-Hep-1 and (C) Huh-7 cells. The cells were counted at the indicated time points following transfection using a trypan blue exclusion assay. The data are presented as the mean \pm standard deviation of 3 independent experiments and the groups were compared using two-way analysis of variance. ${ }^{*} \mathrm{P}<0.05$. (D) OTC downregulation led to enhanced colony formation. Transfected SK-Hep-1 and Huh-7 cells were cultured for 12 days, images were captured, and newly formed colonies in each well were counted and expressed as a percentage relative to the control group. The data are presented as the mean \pm standard deviation of 3 independent experiments. ${ }^{*} \mathrm{P}<0.05,{ }^{* *} \mathrm{P}<0.01$ and ${ }^{* * *} \mathrm{P}<0.001$. DNA synthesis was determined in (E) SK-Hep-1 and (F) Huh-7 cells 3 days after transfection. Original magnification, $\mathrm{x} 400$. The quantification of EdU-positive cells was conducted macroscopically and expressed as a percentage relative to the control cells. The data are presented as the mean \pm standard deviation of 3 independent experiments. ${ }^{*} \mathrm{P}<0.05,{ }^{* * *} \mathrm{P}<0.01$ and ${ }^{* * * *} \mathrm{P}<0.001$. OTC, ornithine transcarbamylase; HCC, hepatocellular carcinoma; siRNA, small interfering RNA; siOTC, siRNA targeting OTC; siCont, scrambled siRNA control.

OTC silencing facilitates HCC cell proliferation. To further evaluate the biological effects of OTC on HCC cells, an RNA interference experiment was performed on Huh-7 and SK-Hep-1 cells. Targeting siRNAs (siOTC-1 and siOTC-2) suppressed OTC expression, as determined by western blotting (Fig. 4A). OTC downregulation significantly enhanced cell proliferation in the two cell lines (Fig. 4B and C), and resulted in enhanced colony-formation ability (Fig. 4D). In addition, elevated DNA synthesis was observed in OTC-knockdown cells (Fig. 4E and F). Together, these data support that OTC downregulation promotes HCC cell proliferation and colony formation.

\section{Discussion}

OTC, a key enzyme in the urea cycle, has been well documented in ammonia metabolism (20). However, few studies have focused on the role of OTC in HCC. A previous study 
demonstrated that OTC deficiency could increase the risk of developing HCC, suggesting that OTC may be associated with HCC progression. Another study revealed that OTC depletion could increase the probability of liver fibrosis in a mouse model, and since $\sim 90 \%$ of HCC develops from liver fibrosis, this suggests that OTC is indirectly linked to HCC progression $(11,12)$. However, direct evidence on the role of OTC in $\mathrm{HCC}$ progression is lacking. In the present study, the functional role of OTC in HCC was investigated. Based on the database analyses, OTC expression levels were revealed to be downregulated in HCC. This was further verified in liver cancer cell lines and 42 paired clinical HCC tissue samples. In agreement with the present findings, Liu et al (21) noticed that OTC mRNA levels are downregulated in HCC Huh-7 and LH86 cell lines. To further elucidate the role of OTC in HCC, associations between OTC expression and the clinicopathological features of the patients were investigated. The results demonstrated that low OTC expression was associated with larger tumor size and advanced grade. Furthermore, low tumor OTC expression indicated poor prognosis for patients with HCC.

The enzyme activity of OTC is inhibited by acetylation at lysine 88 , highlighting the important role of a deacetylase in the regulation of its function (6). Furthermore, the mitochondrial NAD-dependent protein deacetylase sirtuin-3 (SIRT3) has been identified as a regulator of OTC deacetylation (22). Notably, a previous study determined that SIRT3 is downregulated in HCC cells, possibly protecting them from apoptosis (23). Combined with the present observations that OTC functions as a tumor suppressor, it was hypothesized that the acetylation of OTC in HCC cells is increased by the downregulation of SIRT3, leading to the suppression of its activity. This may explain the finding that OTC RNA interference promoted cell proliferation, whereas overexpression had the opposite effect in the present study.

It is worth noting that decreased OTC levels may impair the normal urea cycle, resulting in the accumulation of ammonia and a hyperammonemic state. High concentrations of ammonia in cells are toxic and induce cell apoptosis (24-27). This is contradictory to the observation that low OTC promoted HCC cell proliferation. In the present study, no clinical symptoms of hyperammonemia nor marked elevations of blood ammonia levels were observed in the patients with HCC. As glutamine metabolism is the main source of ammonia, it is hypothesized that HCC cells may utilize glutamine mainly through the transamination pathway without resulting in ammonia production. This non-canonical pathway of glutamine utilization is also observed in human pancreatic ductal adenocarcinoma cells, and it is required for tumor growth (28). The implicated interactions between the transamination and amino acid metabolism pathways in HCC cells may be involved in the development of HCC mediated by downregulated OTC. Additionally, studies have reported that OTC may influence liver regeneration though participating in certain unidentified signaling pathways, independent of its known enzyme activity (29). Further investigations are required to explain whether the effect of OTC on HCC cells relies on the protein itself or its catalytic properties and possible targets.

In summary, the present study revealed a new function of OTC in HCC tumorigenesis and progression. The decreased OTC levels may contribute to the HCC development of this disease, making it a potential target for therapy. Further studies are required to uncover the underlying mechanism involving OTC in the inhibition of HCC development.

\section{Acknowledgements}

Not applicable.

\section{Funding}

This study was supported by the National Natural Science Foundation of China (grant no. 81672012 to JC), the Chongqing Natural Science Foundation (grant no. cstc2016jcyjA0183 to JC) and the Scientific Research Project of Graduate Students of Chongqing Medical University (grant no. BJRC201726).

\section{Availability of data and materials}

All data generated or analyzed during this study are included in this published article.

\section{Authors' contributions}

JC was involved in the study design, data curating, project administration and resource management. JC and LH participated in the data analysis, funding acquisition, and manuscript writing, editing and reviewing. LH, FR, NT, ZZ and JR took part in specimen collection and acquisition of clinicopathological data. FR, NT, ZZ and JR performed the analysis and interpretation of clinicopathological data. $\mathrm{LH}, \mathrm{XC}, \mathrm{HZ}$ and QY performed the experiments. JC, LH, HZ and SC were involved in the analysis of online datasets. All authors read and approved the final manuscript.

\section{Ethics approval and consent to participate}

The Clinical Research Ethics Committee of the Chongqing Medical University approved the study (approval no. 2017019). Written informed consent was obtained from all participants for use of their tissue samples.

\section{Patient consent for publication}

Not applicable.

\section{Competing interests}

The authors declare that there are no competing interests.

\section{References}

1. Torre LA, Bray F, Siegel RL, Ferlay J, Lortet-Tieulent J and Jemal A: Global cancer statistics, 2012. CA Cancer J Clin 65: 87-108, 2015.

2. El-Serag HB: Hepatocellular carcinoma. N Engl J Med 365: 1118-1127, 2011.

3. Bray F, Ferlay J, Soerjomataram I, Siegel RL, Torre LA and Jemal A: Global cancer statistics 2018: GLOBOCAN estimates of incidence and mortality worldwide for 36 cancers in 185 countries. CA Cancer J Clin 68: 394-424, 2018.

4. Forner A, Reig ME, de Lope CR and Bruix J: Current strategy for staging and treatment: The BCLC update and future prospects. Semin Liver Dis 30: 61-74, 2010. 
5. Raza A and Sood GK: Hepatocellular carcinoma review: Current treatment, and evidence-based medicine. World J Gastroenterol 20: 4115-4127, 2014.

6. Yu W, Lin Y, Yao J, Huang W, Lei Q, Xiong Y, Zhao S and Guan KL: Lysine 88 acetylation negatively regulates ornithine carbamoyltransferase activity in response to nutrient signals. J Biol Chem 284: 13669-13675, 2009.

7. Auron A and Brophy PD: Hyperammonemia in review: Pathophysiology, diagnosis, and treatment. Pediatr Nephrol 27 207-222, 2012.

8. Helman G, Pacheco-Colón I and Gropman AL: The urea cycle disorders. Semin Neurol 34: 341-349, 2014.

9. Capistrano-Estrada S, Marsden DL, Nyhan WL, Newbury RO, Krous HF and Tuchman M: Histopathological findings in a male with late-onset ornithine transcarbamylase deficiency. Pediatr Pathol 14: 235-243, 1994.

10. El-Serag HB and Mason AC: Rising incidence of hepatocellular carcinoma in the United States. N Engl J Med 340: 745-750, 1999.

11. Wilson JM, Shchelochkov OA, Gallagher RC and Batshaw ML: Hepatocellular carcinoma in a research subject with ornithine transcarbamylase deficiency. Mol Genet Metab 105: 263-265, 2012.

12. Wang L, Bell P, Morizono H, He Z, Pumbo E, Yu H, White J, Batshaw ML and Wilson JM: AAV gene therapy corrects OTC deficiency and prevents liver fibrosis in aged OTC-knock out heterozygous mice. Mol Genet Metab 120: 299-305, 2017.

13. Cuccurullo V and Mansi L: AJCC cancer staging handbook: From the AJCC cancer staging manual (7th edition). Eur J Nucl Med Mol Imaging 38: 408-408, 2011.

14. Edmondson HA and Steiner PE: Primary carcinoma of the liver: A study of 100 cases among 48,900 necropsies. Cancer 7: 462-503, 1954

15. Dang H, Takai A, Forgues M, Pomyen Y, Mou H, Xue W, Ray D, $\mathrm{Ha} \mathrm{KCH}$, Morris QD, Hughes TR and Wang XW: Oncogenic activation of the RNA binding protein NELFE and MYC signaling in hepatocellular carcinoma. Cancer Cell 32: 101-114 e08, 2017.

16. Livak KJ and Schmittgen TD: Analysis of relative gene expression data using real-time quantitative PCR and the 2(-Delta Delta C(T)) method. Methods 25: 402-408, 2001

17. Chen J, Zhang B, Wong N, Lo AW, To KF, Chan AW, Ng MH, Ho CY, Cheng SH, Lai PB, et al: Sirtuin 1 is upregulated in a subset of hepatocellular carcinomas where it is essential for telomere maintenance and tumor cell growth. Cancer Res 71: 4138-4149, 2011.

18. Tommasi S, Zheng A, Weninger A, Bates SE, Li XA, Wu X, Hollstein $M$ and Besaratinia A: Mammalian cells acquire epigenetic hallmarks of human cancer during immortalization. Nucleic Acids Res 41: 182-195, 2013.

19. Ramboer E, De Craene B, De Kock J, Vanhaecke T, Berx G, Rogiers V and Vinken M: Strategies for immortalization of primary hepatocytes. J Hepatol 61: 925-943, 2014.
20. Mori M, Miura S, Morita T, Takiguchi M and Tatibana M: Ornithine transcarbamylase in liver mitochondria. Mol Cell Biochem 49: 97-111, 1982.

21. Liu H, Dong H, Robertson K and Liu C: DNA methylation suppresses expression of the urea cycle enzyme carbamoyl phosphate synthetase 1 (CPS1) in human hepatocellular carcinoma. Am J Pathol 178: 652-661, 2011.

22. Hallows WC, Yu W, Smith BC, Devries MK, Ellinger JJ, Someya S, Shortreed MR, Prolla T, Markley JL, Smith LM, et al: Sirt3 promotes the urea cycle and fatty acid oxidation during dietary restriction. Mol Cell 41: 139-149, 2011.

23. Song CL, Tang H, Ran LK, Ko BC, Zhang ZZ, Chen X, Ren JH, Tao NN, Li WY, Huang AL and Chen J: Sirtuin 3 inhibits hepatocellular carcinoma growth through the glycogen synthase kinase-3 $\beta$ /BCL2-associated X protein-dependent apoptotic pathway. Oncogene 35: 631-641, 2016.

24. Liu Q, Ding MH, Zhang R, Chen HX, Zhou XX, Xu HF, Chen H and Peng KL: Study on mechanism of thyroid cytotoxicity of ammonium perchlorate. Zhonghua Lao Dong Wei Sheng Zhi Ye Bing Za Zhi 31: 418-421, 2013 (In Chinese).

25. Luo C, Shen G, Liu N, Gong F, Wei X, Yao S, Liu D, Teng X, Ye N, Zhang N, et al: Ammonia drives dendritic cells into dysfunction. J Immunol 193: 1080-1089, 2014.

26. Wang Q, Wang Y, Yu Z, Li D, Jia B, Li J, Guan K, Zhou Y, Chen $Y$ and Kan Q: Ammonia-induced energy disorders interfere with bilirubin metabolism in hepatocytes. Arch Biochem Biophys 555-556: 16-22, 2014.

27. Xu Y, Wang N, Ding Y, Wang C, Yu Y, Liu S, Wang X and Li Z: Ammonium chloride enhances cisplatin cytotoxicity through DNA double-strand breaks in human cervical cancer cells. Oncol Rep 30: 1195-1200, 2013.

28. Son J, Lyssiotis CA, Ying H, Wang X, Hua S, Ligorio M, Perera RM, Ferrone CR, Mullarky E, Shyh-Chang N, et al: Glutamine supports pancreatic cancer growth through a KRAS-regulated metabolic pathway. Nature 496: 101-105, 2013.

29. Díaz-Muñoz M and Hernández-Muñoz R: Molecular and biochemical features of the mitochondrial enzyme ornithine transcarbamylase: A possible new role as a signaling factor. Curr Med Chem 17: 2253-2260, 2010.

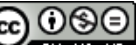

This work is licensed under a Creative Commons Attribution-NonCommercial-NoDerivatives 4.0 International (CC BY-NC-ND 4.0) License. 